\section{Immunosuppression-associated posterior reversible encephalopathy syndrome in an acute leukemia case}

\author{
Umit Y. Malkan, Gursel Gunes, \\ Haluk Demiroglu, Hakan Goker
}

Department of Hematology, Hacettepe University School of Medicine, Ankara, Turkey

\begin{abstract}
Posterior reversible encephalopathy syndrome (PRES) was described in 1996. Herein, we aimed to report an immunosuppression-related PRES case. A 34-year-old woman was diagnosed as t-cell acute lymphoblastic leukemia and allogeneic hematopoietic stem cell transplantation (HSCT) was performed. Cyclosporine was given for GVHD prophylaxis in addition to syndrome of headache, confusion or reduced level of consciousness, visual alterations, and seizures, that was related with characteristic neuroimaging signs of posterior cerebral white matter edema. ${ }^{3}$ PRES is related with different medical conditions such as hypertensive encephalopathy, eclampsia and the use of cytotoxic and immunosuppressant drugs. Herein, we aimed to report a case that had PRES secondary to immunosuppression after HSCT.
\end{abstract} the other routine medications of HSCT. She was hospitalized for acute renal failure and due to the possible contribution of acute renal failure cyclosporine was stopped. Tacrolimus was started for GVHD prophylaxis at a dose of $1 \mathrm{mg} /$ day. However, fifteen days after the initiation of tacrolimus, blurred vision occurred in our patient. Petechial bleeding sites were detected in bilateral cerebral and cerebellar hemisphere by MR imaging. Tacrolimus dosage was reduced to 0.5 $\mathrm{mg} /$ day. She had hypertension which was difficult to control and followed-up in the intensive care unit. She had seizures. Control cranial MR resulted as diffusion limitation in bilateral cerebellar hemisphere, bilateral occipital and frontal-parietal regions with vasogenic edema findings; contrast involvement in left frontal-parietal and right cerebellar regions. She had vision loss and lethargy. Control cranial MR favored PRES syndrome secondary to immunosuppression. Hypertensive state was taken under control with antihypertensive treatment and all immunosuppressive agents were stopped. Two weeks later her clinical condition was slightly improved. MR test which was conducted 2 weeks after the diagnosis revealed the regression of PRES lesions. The characteristic signs on neuroimaging are the symmetrical white matter edema in the posterior cerebral hemispheres, particularly the parietal-occipital regions. In conclusion, PRES rarely develops secondary to the immunosuppressive agents and the clinicians should suspect and promptly diagnose PRES which might cause otherwise serious irreversible clinical complications.

\section{Introduction}

Graft versus host disease (GVHD) is a serious complication that may occur in 30 $50 \%$ patients after allogeneic hematopoietic stem cell transplantation (HSCT). ${ }^{1}$ Cyclosporine and tacrolimus are widely used for immunosuppression for prophylaxis of GVHD in order to prevent this serious complication. $^{2}$ However immunosuppression associated toxicities may develop in some transplanted patients. Posterior reversible encephalopathy syndrome (PRES) was described in 1996 as a clinical

\section{Case Report}

A 34-year-old woman was diagnosed with t-cell acute lymphoblastic leukemia (ALL). She was given induction therapy and achieved complete remission. Then, CALGB early intensification treatment, intratechal methotrexate and consolidation chemotherapy was started. Subsequently, in October 2015, cranial radiotherapy was administered in her lumber punctures, there was no blastic involvement. In October 2015, allogeneic hematopoietic stem cell transplantation (HSCT) was performed from her full match donor. Cyclosporine was started for GVHD prophylaxis with other routine prophylaxis medications of HSCT. In her routine controls, serum creatihospitalized for acute renal failure. Cyclosporine stopped and tacrolimus treatment was started with dose of $1 \mathrm{mg} /$ day. With this treatment modification serum creatinine level was normalized. However, 15 days after the initiation of tacrolimus, blurred vision occurred in this patient. She was consulted to neurology and eye departments. Petechial bleeding sites were detected in bilateral cerebral and cerebellar hemisphere by MR imaging. Enlargement of left retro cerebellar BOS distance was detected by CT. Orbital MR revealed bilateral sub retinal hemorrhages. Transthoracic echocardiography revealed massive pleural effusion. Bone marrow examination revealed normal results and bone marrow chimerism nine was detected as $2.3 \mathrm{mg} / \mathrm{dL}$ and she was
Correspondence: Hakan Goker, Department of Hematology, Hacettepe University School of Medicine, TR-06100, Ankara, Turkey. Tel.: +90.3123053050 - Fax: +90.3123051614 E-mail: hgoker1@yahoo.com

Key words: Posterior reversible encephalopathy syndrome; immunosuppression; leukemia.

Contributions: the authors contributed equally.

Conflict of interest: the authors declare no potential conflict of interest.

Funding: none.

Received for publication: 10 June 2017.

Accepted for publication: 2 May 2018.

This work is licensed under a Creative Commons Attribution-NonCommercial 4.0 International License (CC BY-NC 4.0).

(C) Copyright U.Y. Malkan et al., 2018

Licensee PAGEPress, Italy

Hematology Reports 2018; 10:7257

doi:10.4081/hr.2018.7257

test showed $98 \%$ donor profile. Tacrolimus dosage was reduced to $0.5 \mathrm{mg} /$ day. She had difficulty to control hypertension and followed-up in intensive care unit. She also had seizures. Electroencephalography tests confirm epileptiform activities. She was given levetiracetam $2000 \mathrm{mg}$ /day in order to control seizures. Control cranial MR favored PRES syndrome (Figure 1). She had loss of vision; her mental and clinical condition was deteriorated. She was diagnosed as PRES syndrome secondary to immunosuppression. Hypertensive state was taken under control with antihypertensive treatment and all immunosuppressive agents were stopped. After two weeks her mental and clinical condition improved but still with visual disturbances. The control MR test which was conducted 2 weeks after the diagnosis revealed the regression of PRES lesions compared to previous MR test (Figure 2). The patient is still followedup in our clinic.

\section{Discussion and Conclusions}

The incidence of PRES is not clarified yet. PRES is encountered more in females. ${ }^{4}$ The mechanism of PRES is still unclear, but it appears to be associated with damaged cerebral auto regulation and endothelial dysfunction. ${ }^{3}$ Arteriolar constriction and dilatation in cerebral veins controls the blood flow and in case of dysregulation 


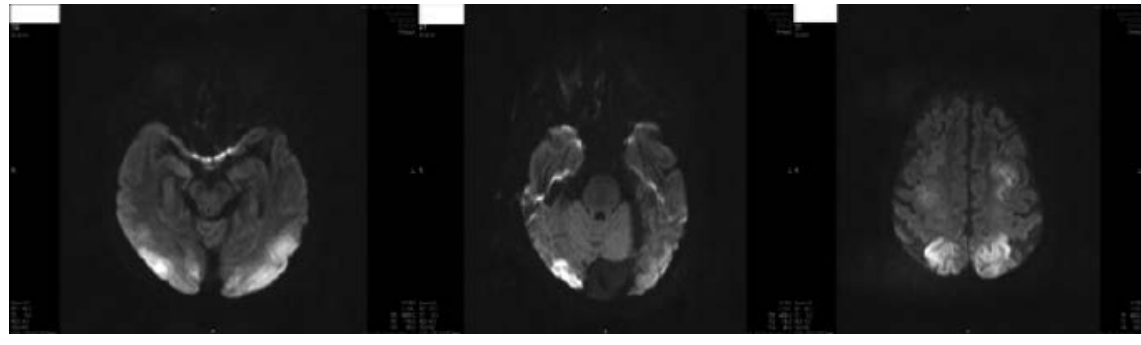

Figure 1. Diffusion limitation in bilateral cerebellar hemisphere, bilateral occipital and frontoparietal regions, before the treatment.

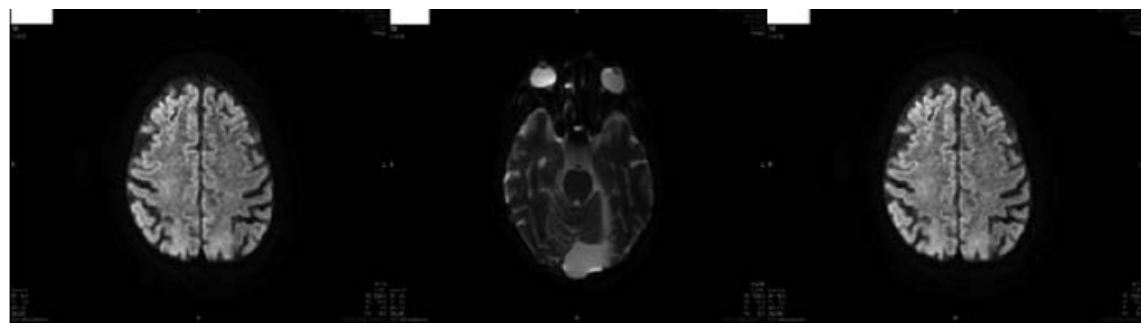

Figure 2. Regression of PRES lesions was observed compared to previous MR screening.

PRES syndrome may develop. ${ }^{5}$ Endothelial dysfunction and cerebral ischemia may also contribute to the process. ${ }^{6,7}$ Hypertension, renal diseases and immunosuppressive treatments are risk factors for PRES. ${ }^{8}$ Cyclosporine and tacrolimus are shown to be related with PRES. ${ }^{9,10}$ The clinical manifestations of PRES is generally present as headaches, altered consciousness, visual disturbances and seizures. ${ }^{11}$ Diagnosis of PRES depends on imaging techniques. The characteristic signs on neuroimaging are the symmetrical white matter edema in the posterior cerebral hemispheres, particularly the parietal-occipital regions. ${ }^{12}$ Prompt recognition and initiation of therapy is significant to prevent the permanent damage which may develop in this otherwise typically reversible condition. Hypertension and seizures should quickly be taken under control. Decrease in drug dosage or prompt discontinuation of the cytotoxic drug is generally recommended in cases of PRES related with cytotoxic agents and is frequently related with clinical improvement. ${ }^{13}$ If the management of patients could be done immediately, the prognosis of PRES cases are generally well. After the dismissal of the predisposing factor and control of the blood pressure, the clinic of PRES cases normalizes in a period of days to weeks. ${ }^{14}$ The knowledge about PRES is somewhat limited because the heterogeneous clinical settings in which PRES develops and clinical reports are limited to case reports and small series. ${ }^{15,16}$ This case is similar to the litera- ture because the PRES was developed in this case due to the immunosuppression. Additionally, the clinical picture of our case was improved in two weeks after the possible diagnosis of PRES. In conclusion, PRES may develop secondary to the immunosuppressive agents and the clinicians should promptly diagnose PRES otherwise serious irreversible clinical complications may possibly occur.

\section{References}

1. Goker H, Ozdemir E, Uz B, et al. Comparative outcome of reduced intensity and myeloablative conditioning regimen in HLA identical sibling allogeneic hematopoietic stem cell transplantation for acute leukemia patients: a single center experience. Transfus Apher Sci 2013;49:590-9.

2. Tekgündüz E, Kaynar L, Göker H, et al. Retrospective analysis of adult patients with acute lymphoblastic leukemia undergoing allogeneic hematopoietic cell transplantation: A multicenter experience of daily practice. Transfus Apher Sci 2016;54:41-7.

3. Hinchey J, Chaves C, Appignani B, et al. A reversible posterior leukoencephalopathy syndrome. N Engl J Med 1996;334:494.

4. Ay H, Buonanno FS, Schaefer PW, et al. Posterior leukoencephalopathy without severe hypertension: utility of diffu- sion-weighted MRI. Neurology 1998;51:1369.

5. Paulson OB, Strandgaard S, Edvinsson L. Cerebral autoregulation. Cerebrovasc Brain Metab Rev 1990;2:161.

6. Mukherjee P, McKinstry RC. Reversible posterior leukoencephalopathy syndrome: evaluation with diffusion-tensor MR imaging. Radiology 2001;219:756.

7. Covarrubias DJ, Luetmer PH, Campeau NG. Posterior reversible encephalopathy syndrome: prognostic utility of quantitative diffusion-weighted MR images. AJNR Am J Neuroradiol 2002; 23:1038.

8. Schwartz RB, Jones KM, Kalina P, et al. Hypertensive encephalopathy: findings on CT, MR imaging, and SPECT imaging in 14 cases. AJR Am J Roentgenol 1992;159:379.

9. Kou R, Greif D, Michel T. Dephosphorylation of endothelial nitric-oxide synthase by vascular endothelial growth factor. Implications for the vascular responses to cyclosporin A. J Biol Chem 2002;277: 29669.

10. Junna MR, Rabinstein AA. Tacrolimus induced leukoencephalopathy presenting with status epilepticus and prolonged coma. J Neurol Neurosurg Psychiatry 2007;78:1410.

11. Fugate JE, Claassen DO, Cloft HJ, et al. Posterior reversible encephalopathy syndrome: associated clinical and radiologic findings. Mayo Clin Proc 2010;85:427.

12. Lamy C, Oppenheim C, Méder JF, Mas JL. Neuroimaging in posterior reversible encephalopathy syndrome. J Neuroimaging 2004;14:89.

13. Hayes D Jr, Adler B, Turner TL, Mansour HM. Alternative tacrolimus and sirolimus regimen associated with rapid resolution of posterior reversible encephalopathy syndrome after lung transplantation. Pediatr Neurol 2014; 50:272.

14. Roth C, Ferbert A. Posterior reversible encephalopathy syndrome: long-term follow-up. J Neurol Neurosurg Psychiatry 2010;81:773.

15. Kamiya-Matsuoka C, Paker AM, Chi L, et al. Posterior reversible encephalopathy syndrome in cancer patients: a single institution retrospective study. J Neurooncol 2016;128:75-84.

16. Aruch DB, Renteria A. Simultaneous PRES and TMA secondary to tacrolimus after allogeneic bone marrow transplant. Blood 2015;125:3963. 